\title{
Power optimization of gas pipelines via an improved particle swarm optimization algorithm
}

\author{
Zheng Zhiwei and Wu Changchun*
}

Beijing Key Laboratory of Urban Oil and Gas Distribution Technology, China University of Petroleum, Beijing 102249, China

(C) China University of Petroleum (Beijing) and Springer-Verlag Berlin Heidelberg 2012

\begin{abstract}
In past decades dynamic programming, genetic algorithms, ant colony optimization algorithms and some gradient algorithms have been applied to power optimization of gas pipelines. In this paper a power optimization model for gas pipelines is developed and an improved particle swarm optimization algorithm is applied. Based on the testing of the parameters involved in the algorithm which need to be defined artificially, the values of these parameters have been recommended which can make the algorithm reach efficiently the approximate optimum solution with required accuracy. Some examples have shown that the relative error of the particle swarm optimization over ant colony optimization and dynamic programming is less than $1 \%$ and the computation time is much less than that of ant colony optimization and dynamic programming.
\end{abstract}

Key words: Gas pipeline, operation, optimization, particle swarm optimization algorithm

\section{Introduction}

Compressors provide the pressure necessary to transport natural gas via a pipeline. The prime mover consumes much power when driving the compressor. So power optimization is very important for improving the operational economy of gas pipelines.

The objective function of power optimization is nonlinear and non-convex. Many constraints are non-linear. It is difficult to solve this kind of optimization problems. Dynamic programming (DP) (Wong and Larson, 1968a; 1968b; Peretti and Toth, 1982; Carter, 1998; Ríos-Mercado et al, 2006), artificial intelligence (Sun et al, 2000) and some gradient algorithms (Percell and Ryan, 1987; Wu et al, 2000) have been applied to power optimization of gas pipelines. These methods are effective. However, the application range of traditional algorithms is limited and the computation time is long. Particle swarm optimization (PSO) algorithm is a colony intelligence calculation technique developed by Kennedy and Eberhart in 1995. It is inspired by the social behavior of organisms such as bird flocking (Yu et al, 2009). The PSO belongs to evolution algorithms. Like genetic algorithm it also begins with some random solutions and searches for the optimum solution by iterative computation. The PSO also evaluates solutions by the fitness function, but it does not have the process of crossover and mutation. The PSO searches for the optimum solution by tracking the best positions of individual and colony after evolution for one

*Corresponding author. email: wuchangchun@vip.sina.com Received April 15, 2011 generation. Because the convergence of PSO is fast and the accuracy is high, the researchers pay much attention to the PSO. It is one of the most popular optimization algorithms presently (Van den Bergh and Engelbrecht, 2006; Liu et al, 2007; Perez and Behdinan, 2007; Chen et al, 2010).

In this paper an improved PSO is introduced. Because the colony information is very important, after evolution for one generation, the best position of the particle swarm will be sought again $n$ times around itself. If a better position can be found, it will be the new best position of the particle swarm. If no better position can be found, the best position of the particle swarm will not be changed. It is good for the algorithm to avoid local solutions effectively and the accuracy can be improved. The improved PSO is applied to the power optimization of gas pipelines. Some parameters of the algorithm are tested in order to get the most fitting values for the power optimization. Some cases show that the computation time can be significantly saved compared to ant colony optimization (ACO) and DP.

\section{Power optimization model of gas pipelines}

In power optimization of a gas pipeline (as shown in Fig. 1), the objective function is minimizing the total energy consumed by all the compressor units and the decision variables are the discharge pressures of compressor stations. The constraints include hydraulic constraints and thermal constraints of gas flow in the pipe, the characteristic equations of compressors, the maximum allowable discharge pressure and the minimum allowable suction pressure of compressor stations, the minimum allowable delivery pressure at the delivery terminal, the flow rate and speed limits of every 
online compressor, etc. The problem can be formulated as follows:

$$
\min F_{\text {cost }}=\left(\sum_{i=1}^{\text {num }} \sum_{k \in J}^{\text {sta }} Q_{i k} \rho_{i} \frac{m}{m-1} R Z_{i} T_{i}\left(\left(\frac{p_{\mathrm{d}}}{p_{\mathrm{s}}}\right)^{\frac{m-1}{m}}-1\right) / \eta_{i k} / \eta_{\mathrm{r}}\right) / E_{\text {min }}
$$

s.t.

$$
\begin{aligned}
& p_{\mathrm{d}}{ }^{2}-p_{\mathrm{e}}{ }^{2}=f Q^{2} \\
& p_{\mathrm{d}} \leq p_{\max } \\
& p_{\min 1} \leq p_{\mathrm{s}} \\
& p_{\min 2} \leq p_{\text {delivery }} \\
& H_{i k}=a_{0}\left(\frac{n_{i k}}{n_{0 i k}}\right)^{2}+a_{1} Q_{i k} \frac{n_{i k}}{n_{0 i k}}+a_{2} Q_{i k}^{2} \\
& \eta_{i k}=b_{0}+b_{1} Q_{i k} \frac{n_{0 i k}}{n_{i k}}+b_{2} Q_{i k}{ }^{2}\left(\frac{n_{0 i k}}{n_{i k}}\right)^{2} \\
& Q_{\min , i k} \leq Q_{i k} \leq Q_{\max , i k} \\
& n_{\min , i k} \leq n_{i k} \leq n_{\max , i k}
\end{aligned}
$$

where num sta represents the number of compressor stations along the pipeline system; $J$ represents the number of online compressor units in each compressor station; $Q$ is the volume flow rate in the pipeline, $\mathrm{m}^{3} / \mathrm{s} ; m$ is the gas adiabatic index; $R$ is the gas constant, $\mathrm{kJ} /(\mathrm{kmol} \cdot \mathrm{K}) ; \rho_{i}$ is the density of the natural gas at the suction condition of station $i, \mathrm{~kg} / \mathrm{m}^{3} ; Z$ is the gas compressibility factor; $T$ is the gas temperature at the suction condition of the stations, $\mathrm{K} ; H_{i k}$ is the adiabatic head, $\mathrm{kJ} / \mathrm{kg} ; \eta_{i k}$ represents the adiabatic efficiency of compressor $k$ in station $i ; \eta_{r}$ represents the efficiency of the gas turbine; $E_{\min }$ is the low calorific value of gas, $\mathrm{kJ} / \mathrm{m}^{3} ; p_{\mathrm{d}}$ and $p_{\mathrm{s}}$ are the discharge pressure and the suction pressure of a compressor station, $\mathrm{Pa} ; p_{\mathrm{e}}$ is the end pressure of a pipe segment between compressor stations, $\mathrm{Pa} ; p_{\text {delivery }}$ is the supply pressure at the delivery terminal, $\mathrm{Pa} ; p_{\max }$ is the maximum allowable discharge pressure of a compressor station, $\mathrm{Pa} ; p_{\min 1}$ is the minimum allowable suction pressure of a compressor station, $\mathrm{Pa} ; p_{\min 2}$ is the minimum allowable delivery pressure at the delivery terminal, $\mathrm{Pa} ; f$ represents the resistance of pipe segments between compressor stations; $a_{0}, a_{1}, a_{2}, b_{0}, b_{1}, b_{2}$ are coefficients of characteristic equations for a centrifugal compressor; $n_{0 i k}$ and $n_{i k}$ represent the rated speed and the actual speed of compressor $k$ in station $i$, respectively, rpm; $n_{\text {min, } i k}$ and $n_{\text {max }, i k}$ are the minimum speed and the maximum speed of compressor $k$ in station $i, \mathrm{rpm} ; Q_{\min , i k}$ and $Q_{\text {max }, i k}$ are the surge flow rate and the stonewall flow rate of compressor $k$ in station $i, \mathrm{~m}^{3} / \mathrm{s}$.

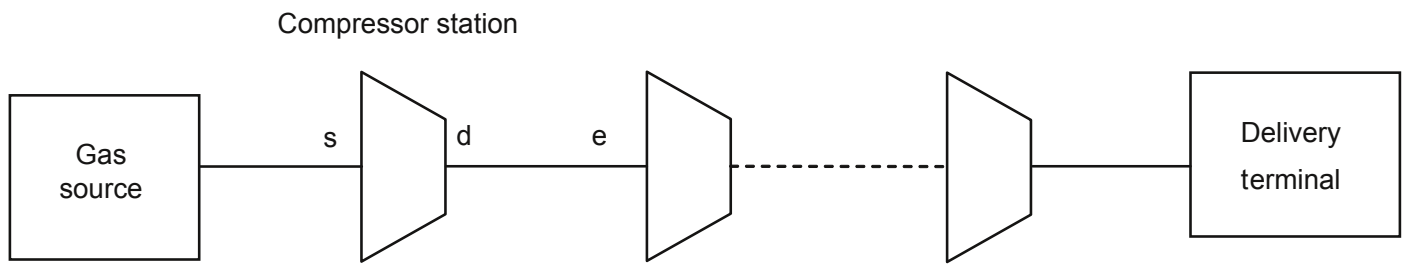

Fig. 1 A gas pipeline system

\section{PSO algorithm}

\subsection{Traditional PSO algorithm}

A particle swarm containing $M$ particles flies in $D$ dimensional space. $x_{i}\left(x_{i 1}, x_{i 2}, x_{i 3}, \cdots, x_{i D}\right)$ represents the position of particle $i$ in the space. $v_{i}\left(v_{i 1}, v_{i 2}, v_{i 3}, \cdots, v_{i D}\right)$ represents the velocity of particle $i . p_{i}\left(p_{i 1}, p_{i 2}, p_{i 3}, \cdots, p_{i D}\right)$ and $p_{g}\left(p_{g 1}, p_{g 2}, p_{g 3}, \cdots, p_{g D}\right)$ are the best positions of particle $i$ and the particle swarm presently. The velocity and position of particle $i$ in the space are adjusted by the following equations (Du and Li, 2008).

$$
v_{i d, t}=w v_{i d, t-1}+c_{1} r_{1}\left(p_{i d, t-1}-x_{i d, t-1}\right)+c_{2} r_{2}\left(p_{g d, t-1}-x_{i d, t-1}\right)
$$

$$
x_{i d, t}=x_{i d, t-1}+v_{i d, t}
$$

where $i=1,2, \cdots, M ; d=1,2, \cdots, D ; t$ represents the evolution generation up to now; $w$ represents the weight factor; $c_{1}$ and $c_{2}$ are the acceleration constants; $r_{1}$ and $r_{2}$ are the random numbers between $[0,1]$.

\subsection{An improved PSO algorithm}

Because the traditional PSO may converge to a local optimum solution and the accuracy is poor (Jiang et al, 2007), an improved PSO is introduced here.

From Eq. (2) it can be seen that the particles are updated by tracking the best positions of individuals and the colony. So the two best positions are very important for the algorithm. The acceleration constants $c_{1}$ and $c_{2}$ represent the biggest step flying to the best positions of individuals and the colony, respectively. The influence of individuals and the colony on the particles is decided by them. If $c_{1}=0$, the particles only have colony experience, the convergence will be very fast and may obtain a local solution. If $c_{2}=0$, the particles do not have colony experiences, the particles fly in the space independently, the optimum solution can not be obtained. The colony information is very important for the algorithm to obtain the global solution. So in the improved PSO after every iterative computation the best position of the particle swarm will be sought again around itself. If a better position can be found, it will be the new best position of the particle swarm. If no better position can be found after searching for $n$ times, the best position of the particle swarm will not be changed. The searching process around $p_{\mathrm{g}}$ is as follows: 


$$
\begin{aligned}
& p_{g d}=p_{g d}(1+(2 \text { rand }-1) m) \\
& m=m_{\text {int }} a+m_{\text {min }} \\
& a=\exp \left(-30 \times\left(\frac{t}{T}\right)^{s}\right)
\end{aligned}
$$

where rand is a random number between $[0,1] ; t$ represents the evolution generation up to now; $T$ represents the set total evolution generations; $S$ belongs to $[1,20] ; m_{\min }$ can be set at $0.01 ; m_{\text {int }}$ will be defined artificially.

In the early stage, $a$ is big, the searching step is large, so the convergence is fast and is good for the algorithm to avoid the local solution. In the late stage, $a$ is small, the searching step is small, the searching around the best position of the colony is accurate, so it is good for the algorithm to increase the optimization accuracy.

\section{Testing about parameters of the algorithm}

The improved PSO is coded by FORTRAN90. Some parameters of the algorithm need to be set artificially and the values of the parameters should be fit for the problems to be solved. A case of gas pipeline power optimization with a simple topological structure is taken to test the parameters.

Fig. 2 is the topological structure of the gas pipeline. The gas pipeline is composed of three compressor stations and one delivery terminal. The gas source pressure is 6.1 $\mathrm{MPa}$ at the suction of station 1 . For every compressor station the maximum allowable discharge pressure is $10 \mathrm{MPa}$, and the minimum allowable suction pressure is $4.7 \mathrm{MPa}$. The minimum allowable delivery pressure is $4 \mathrm{MPa}$ at the end of the pipeline. The design flow rate is $32.7 \times 10^{6} \mathrm{~m}^{3} / \mathrm{d}$. The pipeline is $1,016 \mathrm{~mm}$ in outside diameter, $14.6 \mathrm{~mm}$ in wall thickness and $0.01 \mathrm{~mm}$ in pipe roughness. The objective function is minimizing the total power of all the compressor units and the decision variables are the discharge pressures of all the compressor stations.

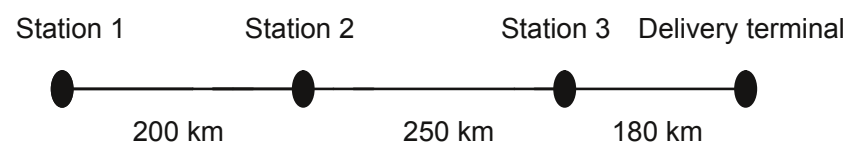

Fig. 2 Topological structure of the gas pipeline

The parameters required to test include $c_{1}, c_{2}, n$, and $m_{\text {int }}$. According to the properties of the algorithm, the best possible value of these parameters can be defined. With $c_{1}, c_{2} \in\{0.2$, $0.4,0.6,0.8,1,2,3\}, n \in\{5,10,20,30,40,50\}, m_{\text {int }} \in\{0.5$, $1,2,3,4,10\}$, the optimal objective function values will be observed to select the best combination of these parameters.

Firstly we can choose $n=40, m_{\text {int }}=1$, when $c_{1}=c_{2}=0.6,0.8$, 1,2 the optimal objective value obtained by the algorithm is smallest, it equals $43.45 \mathrm{MW} . c_{1}=1, c_{2}=1$ can be defined.

With $c_{1}=1, c_{2}=1, m_{\text {int }}=1$, when $n=30,40,50$ the optimal objective value obtained by the algorithm is smallest, it equals 43.45 MW. To pursue the shortest computation time $n=30$ is defined.
With $c_{1}=1, c_{2}=1, n=30$, when $m_{\text {int }}=0.5,1,2,3,4$ the optimal objective value obtained by the algorithm is smallest, it equals $43.45 \mathrm{MW}$.

So the best combination of these parameters can be chosen as $c_{1}=1, c_{2}=1, n=30, m_{\text {int }}=1$.

\section{Case analysis}

Fig. 3 is the topological structure of the gas pipeline. The pipeline is composed of five pipe segments connected in series by five compressor stations and one delivery terminal. The basic data of the pipeline is shown in Table 1 .

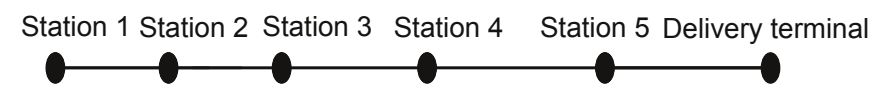

Fig. 3 Compressor stations over the gas pipeline

Table 1 Basic data for the pipeline

\begin{tabular}{cc}
\hline Pipe segment resistances $f$ & $\begin{array}{c}(1.4824,1.7154,1.4613, \\
2.1601,2.3296) \times 10^{8}\end{array}$ \\
\hline Gas constant $R, \mathrm{~kJ} /(\mathrm{kmol} \cdot \mathrm{K})$ & 8.314 \\
\hline Gas source pressure, $\mathrm{MPa}$ & 5 \\
\hline $\begin{array}{c}\text { Maximum allowable discharge } \\
\text { pressure } p_{\max }, \mathrm{MPa}\end{array}$ & 7.2 \\
\hline Minimum allowable suction pressure $p_{\min 1}, \mathrm{MPa}$ & 4.7 \\
\hline Minimum allowable delivery pressure $p_{\min 2}, \mathrm{MPa}$ & 4.2 \\
\hline
\end{tabular}

According to the PSO, the optimal operation scheme for the flow rate of $1.15 \times 10^{6} \mathrm{~kg} / \mathrm{h}$ can be obtained.

Table 2 indicates that the discharge pressures of all stations except Station 5 are 7.2 $\mathrm{MPa}$, the maximum discharge pressure, and the discharge pressure of Station 5 only needs to assure that the pressure at the delivery terminal equals the

\begin{tabular}{|c|c|c|c|c|c|}
\hline \multicolumn{5}{|c|}{ Discharge pressure, $\mathrm{MPa}$} & \multirow{2}{*}{$\begin{array}{c}\text { Total gas consumption } \\
\mathrm{m}^{3} / \mathrm{d}\end{array}$} \\
\hline Station 1 & Station 2 & Station 3 & Station 4 & Station 5 & \\
\hline 7.2 & 7.2 & 7.2 & 7.2 & 6.4 & $3.278 \times 10^{5}$ \\
\hline
\end{tabular}
minimum allowable delivery pressure.

Table 2 Optimal operation scheme

To test the performance of the PSO the optimization results of five flow rates obtained by PSO are compared with ACO and DP.

Table 3 shows for 5 stations the relative error of PSO over $\mathrm{ACO}$ and DP is less than $0.5 \%$, but the computation time is 8 to 9 times less than that of ACO and more than 60 times less than that of DP.

When the stations are increased to 11 , the optimization results of three algorithms are shown in Table 4.

Table 4 shows for 11 stations the relative error of PSO over ACO and DP is less than $1 \%$, but the computation time is 4 to 7 times less than that of $\mathrm{ACO}$ and about 100 times less than that of DP. 
Table 3 The optimization results of three algorithms for 5 stations

\begin{tabular}{ccccccccc}
\hline \multirow{2}{*}{$\begin{array}{c}\text { Flow rate } \\
\mathrm{kg} / \mathrm{h}\end{array}$} & \multicolumn{3}{c}{ Computation time, $\mathrm{s}$} & & \multicolumn{3}{c}{ Gas consumption, } & $10^{5} \mathrm{~m}^{3} / \mathrm{d}$ \\
\cline { 2 - 5 } \cline { 7 - 8 } & PSO & ACO & DP & & PSO & ACO & DP \\
\hline $9.5 \times 10^{5}$ & 24 & 209 & 1727 & & 1.353 & 1.350 & 1.350 \\
$1.00 \times 10^{6}$ & 23 & 203 & 1170 & & 1.849 & 1.853 & 1.852 \\
$1.05 \times 10^{6}$ & 23 & 199 & 1446 & & 2.226 & 2.232 & 2.230 \\
$1.10 \times 10^{6}$ & 23 & 195 & 1544 & & 2.679 & 2.690 & 2.689 \\
$1.15 \times 10^{6}$ & 23 & 185 & 1766 & & 3.278 & 3.283 & 3.282 \\
\hline
\end{tabular}

Table 4 The optimization results of three algorithms for 11 stations

\begin{tabular}{ccccccccc}
\hline \multirow{2}{*}{$\begin{array}{c}\text { Flow rate } \\
\mathrm{kg} / \mathrm{h}\end{array}$} & \multicolumn{3}{c}{ Computation time, $\mathrm{s}$} & & \multicolumn{3}{c}{ Gas consumption, } & $10^{5} \mathrm{~m}^{3} / \mathrm{d}$ \\
\cline { 2 - 4 } \cline { 7 - 8 } & PSO & ACO & DP & & PSO & ACO & DP \\
\hline $9.5 \times 10^{5}$ & 107 & 721 & 10332 & & 3.882 & 3.864 & 3.856 \\
$1.00 \times 10^{6}$ & 106 & 509 & 11533 & & 4.831 & 4.839 & 4.831 \\
$1.05 \times 10^{6}$ & 106 & 477 & 11779 & & 5.801 & 5.764 & 5.751 \\
$1.10 \times 10^{6}$ & 106 & 445 & 11335 & & 6.786 & 6.833 & 6.826 \\
$1.15 \times 10^{6}$ & 106 & 418 & 11150 & & 8.181 & 8.145 & 8.134 \\
\hline
\end{tabular}

When the stations are increased to 17 , the optimization results of three algorithms are shown in Table 5.

Table 5 The optimization results of three algorithms for 17 stations

\begin{tabular}{ccccccccc}
\hline \multirow{2}{*}{$\begin{array}{c}\text { Flow rate } \\
\mathrm{kg} / \mathrm{h}\end{array}$} & \multicolumn{3}{c}{ Computation time, $\mathrm{s}$} & & \multicolumn{3}{c}{ Gas consumption, $10^{5} \mathrm{~m}^{3} / \mathrm{d}$} \\
\cline { 2 - 5 } \cline { 7 - 8 } & PSO & ACO & DP & & PSO & ACO & DP \\
\hline $9.5 \times 10^{5}$ & 166 & 1251 & 28262 & & 6.385 & 6.371 & 6.363 \\
$1.00 \times 10^{6}$ & 163 & 1114 & 30405 & & 7.788 & 7.842 & 7.810 \\
$1.05 \times 10^{6}$ & 162 & 1069 & 29886 & & 9.261 & 9.289 & 9.274 \\
$1.10 \times 10^{6}$ & 163 & 977 & 28560 & & 10.958 & 11.010 & 10.968 \\
$1.15 \times 10^{6}$ & 162 & 649 & 27522 & & 12.940 & 13.024 & 12.958 \\
\hline
\end{tabular}

Table 5 shows for 17 stations the relative error of PSO over ACO and DP is less than $1 \%$, but the computation time is 4 to 8 times less than that of ACO and 170 times less than that of DP.

\section{Conclusions}

In this paper an improved PSO algorithm is applied to gas pipeline power optimization. The optimization results obtained by the algorithm differ from those from ACO and DP by less than $1 \%$, but the computation time can be saved greatly compared with ACO and DP. This will enable us to design a fast and effective decision aid tool to assist operators to make appropriate decisions within a shorter time. For different kinds of optimization problems the parameters involved in the algorithm need to be tested, the values adopted are those that can get better results. This research encourages us to apply PSO to gas network optimization and other difficult optimization problems.

\section{References}

Carter R G. Pipeline optimization: Dynamic programming after 30 years. In: 30th Annual Meeting Pipeline Simulation Interest Group (PSIG), 28-30 October, Denver, Colorado, 1998

Chen M R, Li X, Zhang X, et al. A novel particle swarm optimizer hybridized with extremal optimization. Applied Soft Computing. 2010. 10(2): 367-373

$\mathrm{Du}$ W L and Li B. Multi-strategy ensemble particle swarm optimization for dynamic optimization. Information Sciences. 2008. 178: 30963109

Jiang Y, Hu T S, Huang C C, et al. An improved particle swarm optimization algorithm. Applied Mathematics and Computation. 2007. 193: 231-239

Liu X Y, Liu H and Duan H C. Particle swarm optimization based on dynamic niche technology with applications to conceptual design. Advances in Engineering Software. 2007. 38(10): 668-676

Percell P B and Ryan M J. Steady state optimization of gas pipeline network operation. In: Proceedings of the 19th PSIG annual meeting, Tulsa, October, 1987

Peretti A and Toth P. Optimization of a pipeline for the natural gas transport. European Journal of Operational Research. 1982. 11: 24754

Perez R E and Behdinan K. Particle swarm approach for structural design optimization. Computers and Structures. 2007. 85: 1579-1588

Ríos-Mercado R Z, Kim S and Boyd E A. Efficient operation of natural gas transmission systems: A network-based heuristic for cyclic structures. Computers \& Operations Research. 2006. 33: 2323-2351

Sun C K, Uraikul V, Chan C W, et al. An integrated expert system/ operations research approach for the optimization of natural gas pipeline operations. Engineering Applications of Artificial Intelligence. 2000. 13: 465-475

Van den Bergh F and Engelbrecht A P. A study of particle swarm optimization particle trajectories. Information Sciences. 2006. 176: 937-971

Wong P J and Larson R E. Optimization of natural gas pipeline system via dynamic programming. IEEE Transactions on Automatic Control. 1968a. 5(AC-13): 475-81

Wong P J and Larson R E. Optimization of tree structured natural gas transmission networks. Journal of Mathematical Analysis and Applications. 1968b. 24(3): 613-26

Wu S, Ríos-Mercado R Z, Boyd E A, et al. Model relaxation for the fuel cost minimization of steady state gas pipeline networks. Mathematical and Computer Modelling. 2000. 31: 197-220

Yu X Z, Wei Z and Hai T Z. A modified particle swarm optimization via particle visual modeling analysis. Computers and Mathematics with Applications. 2009. 57: 2022-2029

(Edited by Sun Yanhua) 